\title{
Prévenir et traiter la fracture des instruments endodontiques en NiTi (Nickel-Titane)
}

\section{RÉSUMÉ}

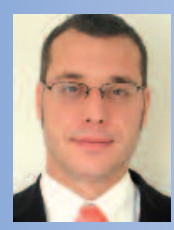

Arnaud EBLE

Chirurgien-dentise libéral, diplômé de la faculté de Nancy, titulaire du CES de biomatériaux appliqués à l'odontologie,

94, rue de la République, 70600 Champlitte, France.
La fracture des instruments endodontiques en NiTi (Nickel-Titane) représente un risque majeur lors du traitement canalaire. L'utilisation des instruments rotatifs demande une connaissance importante aussi bien en théorie qu'en pratique. Le praticien doit maîtriser les propriétés et les limites des instruments utilisés, et doit également adapter aux cas cliniques le système d'instruments rotatifs approprié. Le docteur P. Di Fiore a exposé dans un article douze mesures de prévention. Le respect de ces moyens de prévention n'aboutit pas à l'élimination de la fracture pour autant. Lors de la survenue de cette dernière, il existe différentes techniques de retrait du fragment instrumental. 


\section{Introduction}

$>$

Le traitement endodontique par rota-

tion continue impose aux praticiens une connaissance pointue des bases théoriques et pratiques des instruments en Nickel-Titane. Des mesures de prévention peuvent alors en être déduites. La rotation dans le sens horaire d'un instrument en Nickel-Titane est indispensable $[2,14]$. Il est reconnu par tous les auteurs que la géométrie de la section instrumentale reste un facteur déterminant pour la flexibilité, le taux de défauts, la capacité de déformation plastique et la résistance à la fracture de l'instrument $[19,20,29]$. La réelle contribution des contraintes de torsion et de la fatigue en flexion dans le processus de rupture peut dépendre de la géométrie, de la section de l'instrument et de la distribution des forces sur ce même instrument $[6,9,21,27,29]$. La durée de vie des instruments est inversement proportionnelle à leur taille [3, 7, 10, 15, 22-24]. II existerait même une relation linéaire entre le torque à la fracture et le diamètre instrumental [29]. La rupture en torsion a lieu quand la pointe instrumentale ou tout autre partie de I'instrument se trouve verrouillée dans le canal alors que le moteur continue sa rotation. La limite élastique du métal est dépassée, l'instrument subit une déformation plastique puis la rupture survient [23]. La fracture par le phénomène de torsion apparaît lorsque l'instrument endodontique est soumis à l'effet «taper-lock » [21]. L'autre type de fracture instrumentale est provoqué par le « work-hardening " qui se traduit par la fatigue du métal et une fracture à la flexion. Lors de ce type d'incident, l'instrumentation est en rotation libre dans un canal courbe. Dans la courbure, il se fléchit jusqu'à ce que la fracture se produise au point de flexion maximale [23]. En clinique, la fracture reste une conséquence de ces forces combinées et " hybrides » [17, 27]. L'électropolissage a des effets prolongateurs sur la fatigue de l'instrument grâce à une réduction des irrégularités de surface. Ces irrégularités servent de point de concentration du stress et d'initiation de fissure lors de l'action de l'instrument [1].

\section{Respect des contraintes anatomiques}

\section{Radiographie}

La prise de radiographie est indispensable pour évaluer I'anatomie de la dent. Une radiographie préopératoire est primordiale pour connaître la hauteur de la chambre pulpaire et le nombre de canaux de la dent. La radiographie avec l'instrument en place permet d'établir la longueur de travail. Bien que nécessaire, la radiographie doit être considérée avec plus de recul que d'ordinaire. La prise d'un second cliché radiographique est une précaution supplémentaire au bon déroulement de la préparation canalaire. Par conséquent, la détection de la courbure radiculaire est délicate. Elle inclut l'angle de la courbure, sa direction et sa longueur. Le praticien doit utiliser sa sensation tactile pour déceler la constriction apicale. Mais la courbure radiculaire pourrait rester invisible aux yeux du chirurgien-dentiste [4]. 


\section{Cavité d'accès}

La mise en forme de la cavité d'accès suit différentes phases permettant la trépanation et l'évidement du contenu de la chambre pulpaire [2]. On en dénombre trois, toujours identiques quelle que soit la dent.

\section{> Phase de pénétration}

Elle découvre la chambre pulpaire en réalisant un accès par le plafond pulpaire.

\section{> Phase d'élargissement}

Elle va donner naissance à la forme définitive de la cavité d'accès. Cette phase vient compléter la phase de pénétration, et l'action de la fraise est appliquée sur la paroi dentinaire pour éliminer les derniers débris de la chambre pulpaire. Ainsi, tous les petits angles dentinaires résiduels sont éliminés.

\section{> Phase de finition}

Elle permet de terminer le travail entamé lors des phases précédentes et de polir les murs de la cavité d'accès pour que la transition entre la cavité d'accès et la chambre pulpaire se fasse sans butées.

L'objectif d'une cavité d'accès idéale est de permettre un parage et surtout une progression des instruments sans contraintes ni interférences. Les interférences rencontrées par l'instrument en NiTi augmentent les risques de fracture par fatigue. Selon l'orientation des entrées canalaires, la cavité d'accès peut être plus ou moins retouchée. Le soin endodontique par rotation continue n'en sera que plus simple et se fera en toute sécurité lorsque la cavité d'accès est idéalement préparée.

\section{Préparation des entrées canalaires}

Les forets Gates-Glidden sont les instruments universellement utilisés pour les traitements endodontiques mécanisés. Ces instruments sont reconnus pour leur capacité à évaser la partie coronaire du canal. Ils améliorent ainsi l'accès aux instruments. Cependant certains systèmes sont équipés de limes rotatives dévolues à cet élargissement coronaire. L'alternance lime manuelle/lime mécanisée est également nécessaire pour un évasement suffisant de l'entrée canalaire, et cette précaution est d'autant plus indispensable que le diamètre de l'entrée canalaire est réduit [8].

\section{Amélioration de la cavité d'accès}

En prenant en considération les impératifs de l'accessibilité aux canaux, le praticien peut se poser la question de la conservation d'un pan dentinaire. Il est établi que l'amélioration de la cavité d'accès permet d'obtenir un accès en ligne directe de l'instrument depuis l'entrée coronaire jusqu'à l'apex [5]. Ce trajet en ligne droite limite les contraintes sur l'instrument et par conséquent les risques de fractures. L'obtention des quatre murs permet de conserver un repère pour l'établissement de la longueur de travail et de maintenir les irrigants canalaires en place. Malgré l'amélioration et l'élargissement coronaire des entrées canalaires, la lime peut parfois être confrontée à des courbures qui mettent à mal la propriété de flexibilité de I'instrument. Ces contraintes supplémentaires décuplent le risque de fracture. Le praticien est alors confronté à un choix délicat : soit privilégier le maintien des irrigants canalaires et le repère fourni par les pans de la dent pour la longueur de travail, soit provoquer le délabrement volontaire d'un mur de la dent pour permettre d'une part une insertion de la lime en limitant les contraintes sur cette dernière et d'autre part une augmentation de la visibilité. La notion de bénéfice/risque est alors engagée. Il apparaît clairement que la limitation du 
risque de fracture prend le pas sur la conservation d'un pan dentaire. Le praticien doit alors engager une procédure de délabrement volontaire de la dent malgré ses convictions.

\section{Respect des impératifs instrumentaux}

\section{Vitesse adaptée \\ aux instruments}

La corrélation entre l'augmentation de la vitesse et la majoration des distorsions ou fractures s'explique par le contact entre la lime et les parois canalaires [9]. Ce contact peut entraîner suffisamment de stress pour provoquer la fracture instrumentale. Le fait d'augmenter la vitesse revient à aggraver ces frottements. L'utilisation des instruments de rotation continue à des vitesses faibles diminue sensiblement le risque de fracture de l'instrument $[13,26,28$, 30]. Le respect de la vitesse d'utilisation des limes recommandée par le fabricant permet de doubler son efficacité en termes de durée de préparation et aussi de diviser par 2 le taux de déformation [12]. La vitesse de rotation des limes endodontiques en NiTi dépend d'un compromis à trouver entre l'efficacité de coupe et le risque de fractures. Il faut donc privilégier le premier et minimiser le second.

\section{Technique de préparation canalaire}

La technique du Crown Down est une séquence corono-apicale. Elle commence au niveau du tiers coronaire du canal radiculaire par l'utilisation des instruments de plus gros calibre. Après cette séquence instrumentale du tiers coronaire, des limes de plus en plus fines sont utili-

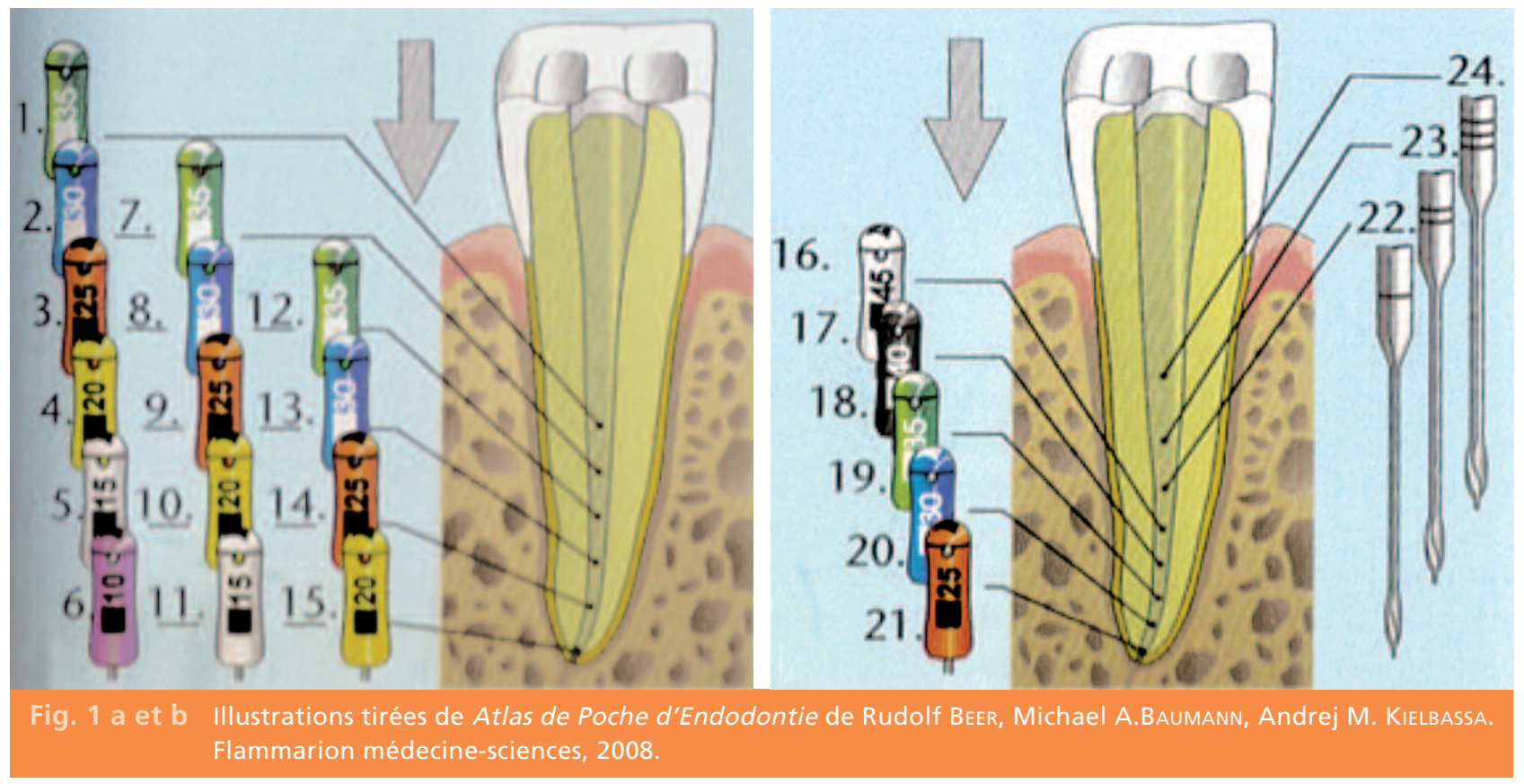


sées de plus en plus profondément dans le canal jusqu'à ce que la plus fine atteigne l'apex radiculaire. Cependant une lime $\mathrm{K}$ numéro 15 est à chaque fois utilisée pour vérifier la perméabilité canalaire sur toute la longueur du canal [4] (fig. 1).

Les avantages de cette technique Crown-Down sont les suivants :

- élimination de la dentine cervicale qui provoque des constrictions canalaires donc des risques de blocage de l'instrument ;

- diminution des courbures canalaires donc diminution des forces de flexion agissant sur l'instrument ;

- pénétration plus profonde, plus rapide et plus massive de la solution de désinfection de type hypochlorite de sodium dans les deux premiers tiers canalaires ;

- élimination de la majeure partie de la pulpe et des débris nécrotiques ou bactériens avant I'approche du tiers apical et donc réduction du risque de repousser des irritants bactériens ou pulpaires dans l'espace péri-apical de réparation ;

- longueur de travail peu ou non modifiée lors de l'instrumentation canalaire puisque la courbure canalaire a été réduite avant l'établissement de la longueur de préparation.

\section{Impact de l'irrigation canalaire}

L'instrument en Nickel-Titane possède une forte résistance à la corrosion. Darabara et al. montrent également que la propagation des microcracks peut être accélérée par l'action combinée du stress subi par l'instrument et du phénomène de corrosion dû à la solution d'irrigation [11]. Berutti et al. décrivent le phénomène d'engainement de la pointe de l'instru- ment qui est à l'origine des fractures de torsion. En effet, cette accumulation de débris dentinaires dans le canal risque de provoquer des bouchons dentinaires mais aussi de verrouiller l'instrument dans le canal et d'aboutir à la fracture. Le nettoyage de l'instrument après chaque utilisation dans un canal est donc essentiel pour limiter ce risque [7].

\section{Les mesures de prévention de P. Di Fiore}

Ces mesures sont proposées par le Dr P. Di Fiore dans un article paru en 2007. L'auteur se base sur dix points réunis lors du traitement endodontique en rotation continue. Les alliages en NiTi présentent des propriétés de superélasticité, de biocompatibilité et de flexion qui rendent le soin plus confortable. Cependant, la fracture instrumentale est un phénomène multifactoriel et complexe. Onze mesures pour prévenir le risque de fractures sont énoncées par l'auteur :

- éviter de soumettre les instruments en NiTi à un stress excessif ;

- utiliser des instruments le moins possible sujets à la fracture ;

- suivre un protocole instrumental précis ;

- détecter l'anatomie canalaire et instrumenter les canaux prudemment ;

- s'assurer que l'accès endodontique est adéquat ;

- ouvrir les orifices canalaires avant de préparer le canal ;

- évaser le canal à l'aide de petites limes manuelles ;

- utiliser les instruments à une vitesse faible et un niveau de Torque faible ;

- préparer le canal en appliquant la technique du Crown-Down ; 
- manipuler les instruments avec un mouvement dynamique de va-et-vient ;

- pour les praticiens inexpérimentés, s'entraîner sur des dents extraites avant I'application clinique.

\section{Traitement de la fracture instrumentale}

Le respect des différents moyens de prévention de la fracture n'évite pas à l'instrument endodontique de se rompre lorsqu'il se retrouve engainé dans le canal dentaire. En raison de leur profil et de l'anatomie canalaire, les instruments de préparation canalaire ne sont jamais liés aux parois dans les trois dimensions. Plusieurs techniques de retrait sont alors envisageables.

\section{> Technique du ByPass}

La technique du ByPass consiste à passer à l'aide d'un instrument endodontique de petit diamètre au-delà du fragment instrumental. Une fois dépassé, le fragment est déverrouillé grâce à l'élargissement du canal [19].

\section{> Instrumentations ultrasonores}

Le dégagement de la portion engainée à l'aide d'inserts abrasifs à faible puissance et la vibration de l'instrument à l'aide d'inserts lisses de martèlement permettent en règle générale son élimination. Les instruments en Nickel-Titane ont tendance à se fracturer avec les vibrations de I'instrument ultrasonore. Les inserts ultrasonores doivent être utilisés avec beaucoup de précaution [16].

\section{> Trousse Masserann}

Les instruments Masserann étant droits, un accès droit et direct est impératif. Le protocole opératoire (fig. 2) distingue trois étapes :

- ouverture de l'accès par élargissement de l'entrée canalaire ;

- dégagement du fragment grâce à l'utilisation dans le sens anti-horaire de forets Trépans ;

- préhension et extraction du fragment en rotation en introduisant la pince d'extraction ouverte jusqu'à la partie dégagée du fragment.

Deux radiographies prises sous différents angles permettent au praticien de contrôler l'orientation du fragment, avant et au moment de l'insertion de la pince d'extraction.

\section{> Amélioration des techniques de retrait}

La combinaison d'instruments automatisés et des ultrasons permet d'extraire les éclats d'instruments verrouillés dans le canal. L'éviction d'un

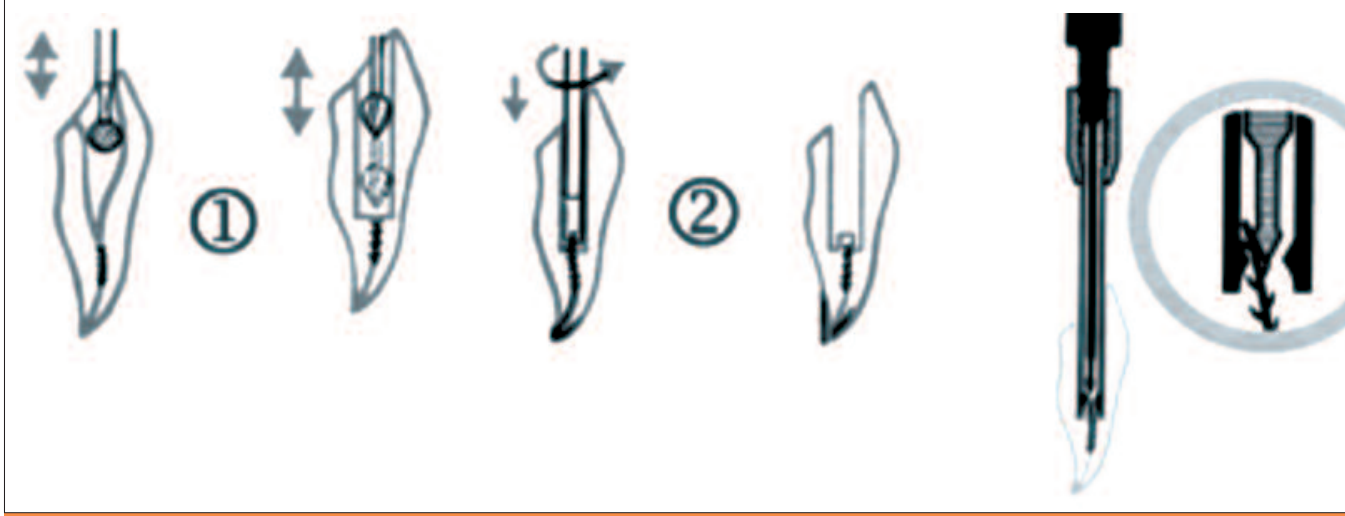

Fig. 2 Protocole d'utilisation du kit Masserann. Illustration tirée de la brochure MicroMega ${ }^{\mathrm{TM}}$. 
élément fracturé peut être améliorée par l'association de deux ou plusieurs dispositifs [18].

Par conséquent, il est nécessaire d'évaluer :

- la courbure canalaire ;

- la position de la dent sur l'arcade ;

- la situation de l'instrument dans le canal dentaire (avant ou après la courbure radiculaire) ;

- la longueur du fragment instrumental ;

- le profil de l'instrument fracturé [25].

\section{Conclusion}

La fracture instrumentale est un incident iatrogène qui complique et compromet le traitement endodontique. L'utilisation quotidienne des instruments en Nickel-Titane nécessite un respect des mesures indiquées ci-dessus. Une étude de laboratoire a indiqué que lorsque le praticien est conscient d'une possible fracture instrumentale et connaît les mesures de prévention de cette fracture, leur incidence est d'environ $0,1 \%$ soit 1 pour 1000 [14]. La fracture des instruments endodontiques en NiTi constitue par conséquent un phénomène com-
Ces paramètres conditionnent l'issue de la tentative de retrait d'un instrument fracturé. Entreprendre ce type de procédure demande un examen minutieux et une localisation précise du site d'intervention.

L'opérateur doit établir le rapport bénéfices/risques de l'intervention dont l'issue est aléatoire. Le praticien doit juger avec beaucoup de précaution la nécessité de ce type d'intervention avant d'entamer le soin.

plexe et multifactoriel. En toute connaissance de cause, le chirurgien-dentiste doit mémoriser ces bases pour anticiper une fracture instrumentale. Lorsque cette fracture survient, le retrait du fragment instrumental est envisageable mais les différentes techniques de retrait sont délicates à entreprendre. Le praticien est alors seul juge de la nécessité de l'acte. Retenons toutefois que lors d'une fracture instrumentale, au-delà de la survenue de cette fracture, son impact sur la santé du patient prime sur tout autre événement.

\section{Bibliographie}

1. Anderson ME, Price JW, Parashos $\mathrm{P}$.

Fracture resistance of electropolished rotary nickel-titanium endodontic instruments.

J Endod 2007;33(10):1212-6.

2. Arbab-Chirani R, Chevalier V, Arbab-Chirani S, Calloch S. Instrumentation canalaire de préparation.
EMC (Elsevier Masson SAS, Paris), Médecine buccale 2010;28-725-A-10.

3. Bahia MG, Buono VT. Decrease in the fatigue resistance of nickel-titanium rotary instruments after clinical use in curved root canals. Oral Surg Oral Med Oral Pathol Oral Radiol Endod
2005;100(2):249-55.

4. Beer $R$, Baumann MA, Kielbassa AM.

Atlas de poche d'endodontie. Paris : Flammarion, Médecine-Sciences, 2006.

5. Bertrand MF.

Anatomie canalaire et radiculaire. Cours Diplôme 
Inter Universitaire d'Endodontie, 2005.

6. Berutti E, Chiandussi G, Gaviglio I, Ibba A.

Comparative analysis of torsional and bending stresses in two mathematical models of nickel-titanium rotary instruments: Protaper versus Profile. J Endod 2003;29(1):15-19.

7. Berutti E, Negro AR, Lendini M, Pasqualini D. Influence of manual preflaring and torque on the failure rate of ProTaper rotary instruments. J Endod 2004;30(4):228-30.

8. Blum JY, Machtou P, Ruddle C, Micallef JP. Analysis of mechanical preparations in extracted teeth using ProTaper rotary instruments: value of the safety quotient. J Endod 2003;29(9):567-75.

9. Claisse A, Haikel $Y$. Les dossiers de l'ADF. Les systèmes Nickel-Titane en rotation continue. Paris : ADF éditions, 2003.

10. Chaves Craveiro de Melo M, Guiomar de Azevedo Bahia M, Lopes Buono VT.

Fatigue resistance of engine-driven rotary nickel-titanium endodontic instruments.

J Endod 2002;28(11):765-9.

11. Darabara $M$, Bourithis $L$, Zinelis S, Papadimitriou GD. Susceptibility to localized corrosion of stainless steel and NiTi endodontic instruments in irrigating solutions. Int Endod J 2004;37(10):705-10.
12. Daugherty DW, Gound TG, Comer TL.

Comparison of fracture rate, deformation rate, and efficiency between rotary endodontic instruments driven at $150 \mathrm{rpm}$ and $350 \mathrm{rpm}$. J Endod 2001;27(2):93-5.

13. Dietz DB, Di Fiore PM, Bahcall JK, Lautenschlager EP. Effect of rational speed on the breakage of nickel-titanium rotary files. J Endod 2000;26(2):68-71.

14. Di Fiore PM.

A dozen ways to prevent nickel-titanium rotary instrument fracture. J Am Dent Assoc 2007;138(2):196-201.

15. Fife D, Gambarini G, Britto LrL.

Cyclic fatigue testing of ProTaper NiTi rotary instruments after clinical use. Oral Surg Oral Med Oral Pathol Oral Radiol Endod 2004;97(2):251-6.

16. Gagnot $\mathrm{G}$.

Les ultrasons

en odontologie.

Rueil-Malmaison : CdP, Coll. Memento, 2008.

17. Gambarini G. Cyclic fatigue of nickel-titanium rotary instruments after clinical use with lowand high-torque endodontic motors.

$J$ Endod 2001;27(12):772-4.

18. Gencoglu N, Helvacioglu D. Comparison of the different techniques to remove fractured endodontic instrument from root canal systems. Eur J Dent 2009;3(2):90-95.
19. Oiknine M, Benizri J. Origine des fractures et de l'usure des limes NiTi en endodontie. Rev Odonto Stomatol 2007;36(2):109-22.

20. Parashos P, Gordon I, Messer $\mathrm{HH}$.

Factors influencing defects of rotary nickel-titanium endodontic instruments after clinical use. J Endod 2004;30(10):722-5.

21. Peters $\mathrm{OA}$, Peters $\mathrm{Cl}$, Schonenberger $\mathrm{K}$, Barbakow F. ProTaper rotary root canal preparation: assessment of torque and force in relation to canal anatomy. Int Endod J 2003;36(2):93-9.

22. Pruett JP, Clement DJ, Carnes DL.

Cyclic fatigue testing of nickel-titanium endodontic instruments. J Endod 1997;23(2):77-85.

23. Sattapan B, Palamara JE, Messer $\mathrm{HH}$.

Torque during canal instrumentation using rotary nickel-titanium files. J Endod 2000;26(3):156-60.

24. Schrader C, Peters OA. Analysis of torque and force with differently tapered rotary endodontic instruments in vitro. J Endod 2005;31(2):120-3.

25. Shen Y, Peng B, Cheung GS. Factors associated with the removal of fractured NiTi instruments from root canal systems. Oral Surg Oral Med Oral Pathol Oral Radiol Endod 2004;98(5):605-10.

26. Li UM, Lee BS, Shih CT, Lan WH, Lin CP. 
Cyclic fatigue of endodontic nickel-titanium rotary instruments: static and dynamic tests. $J$ Endod 2002;28(6):448-51.

27. Ullmann CJ, Peters OA. Effect of cyclic fatigue on static fracture loads in ProTaper nickel-titanium rotary instruments. J Endod 2005;31(3):183-6.
28. Yared GM, Bou Dagher FE, Machtou P.

Failure of ProFile

instruments used with high and low torque motors. Int Endod J 2001;34(6):471-5.

29. Yared GM, Kulkarini GK, Ghossayn F. An in vitro study of the torsionnal properties of new and used K3 instruments.

Int Endod J

2003;36(11):764-9.

30. Zelada G, Varela P, Martin B, Bahillo JG, Magán F, Ahn S.

The effect of rotational speed and the curvature of root canals on the breakage of rotary endodontic instruments. J Endod 2002;28(7):540-2.

\section{SUMMARY}

\section{Preventing and resolving the fracture of endodontic instruments in $\mathrm{NiTi}$}

Arnaud EBLE

Keywords

- root canal treatment

- endodontic

NiTi instruments

- prevention

- fracture
Whenever a dentist performs a root canal treatment, the endodontic nickeltitanium instruments are prone to failure. In order to utilize the rotary instruments safely, one must have an extensive theoretical knowledge of them. It also requires a fair bit of know-how. A good endodontist knows the properties of the instruments he utilizes. He also must comply with their limit, take the clinical case into account and choose the type of rotary instruments accordingly. In an article, Doctor P. di Fiore outlined a 12-point guideline to prevent the endodontic instruments from breaking. The respect of these means of prevention did not lead to the elimination of the failure. In the occurrence of the latter, different techniques for withdrawal of the instrumental fragment can be employed.

\section{Questions-Réponses}

\section{$>$ Quelles sont les 2 types de fractures instrumentales ?}

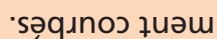

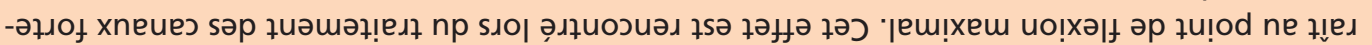
-edde әлпэе

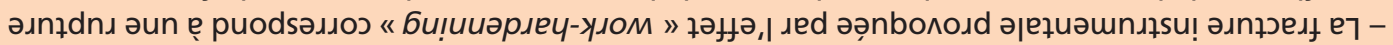

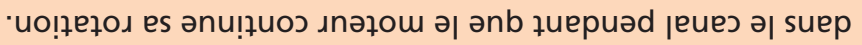

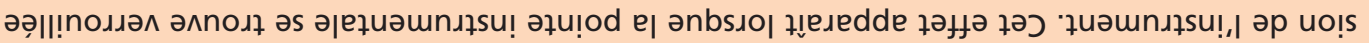

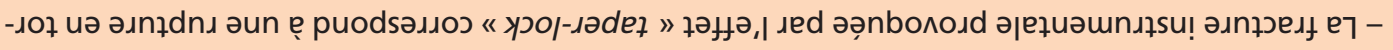


> Quelles sont les phases de réalisation de la cavité d'accès ?

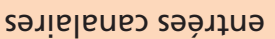

xne sə्गวе,

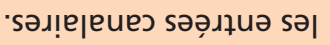

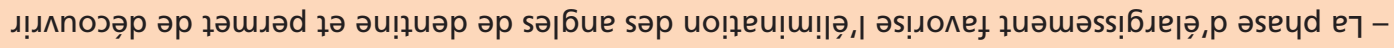
•ə⿱亠䒑e\zh19

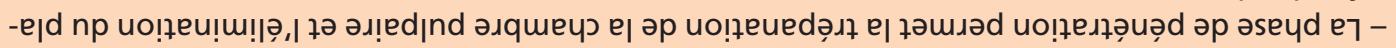

> Les radiographies pré-opératoire, lime en place, cône en place et post-opératoire sont indispensables au traitement endodontique.

$\square$ Vrai Faux

$I \forall y \wedge$ əsuodọy

> Il est préférable de réaliser une radiographie excentrée en mésial ou en distal de la dent traitée pour détecter un maximum de renseignements canalaires.

$I \forall Y \wedge$ əsuodọy

> Quelles sont les techniques de retrait d'un bris instrumental ?

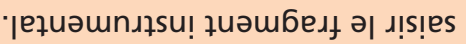

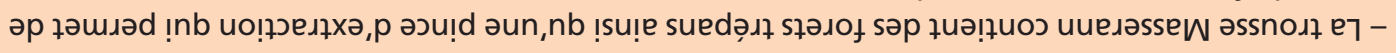
"ұиәшәрінеш әр дәsu!,p suo!?

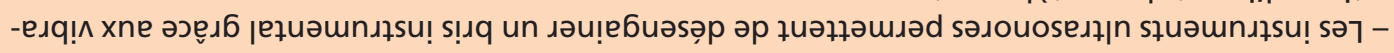

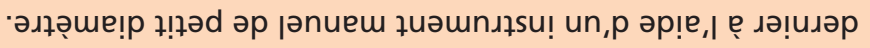

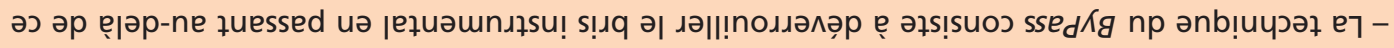

